\title{
Marching cubes technique for volumetric visualization accelerated with graphics processing units
}

\author{
Marcos Vinicius Mussel Cirne · Hélio Pedrini
}

Received: 4 April 2012 / Accepted: 6 December 2012 / Published online: 18 December 2012

(C) The Brazilian Computer Society 2012

\begin{abstract}
Volume visualization has numerous applications that benefit different knowledge domains, such as biology, medicine, meteorology, oceanography, geology, among others. With the continuous advances of technology, it has been possible to achieve considerable rendering rates and a high degree of realism. Visualization tools have currently assisted users with the visual analysis of complex and large datasets. Marching cubes is one of the most widely used real-time volume rendering methods. This paper describes a methodology for speeding up the marching cubes algorithm on a graphics processing unit and discusses a number of ways to improve its performance by means of auxiliary spatial data structures. Experiments conducted with use of several volumetric datasets demonstrate the effectiveness of the developed method.
\end{abstract}

Keywords Volume rendering - Marching cubes .

Volumetric data $\cdot$ Isosurface extraction .

Graphics processing unit

\section{Introduction}

Volume visualization techniques have allowed users to explore and analyze complex data in several domains of knowledge, such as medicine, geology, oceanography, meteorology, biology, among others. Visualization tools provide functionalities for manipulating and rendering volumetric

\footnotetext{
M. V. M. Cirne · H. Pedrini $(\bowtie)$

Institute of Computing, University of Campinas, Campinas, SP13083-852, Brazil

e-mail: helio@ic.unicamp.br

M. V. M. Cirne

e-mail: marcosvcirne@gmail.com
}

datasets, improving the visual comprehension of their structures or patterns.

The development of efficient algorithms for representing, manipulating and rendering complex and large datasets is a challenge of volume visualization. Despite the substantial advances in the field, the use of a central processing unit (CPU) to perform general purpose graphics processing tasks has not been enough to provide effective interactivity or real-time rendering, especially when employed in very large datasets. The constant technological progress enabled the emergence of powerful graphics processing units (GPUs), capable of rendering complex three-dimensional models at a high degree of realism.

The capability of GPUs have been impelled by their high level of parallelism and their ability to perform geometric primitive and floating point operations in a fast and efficient way. GPUs have been recently used for acceleration of various applications, such as fluid dynamics simulation, seismic analysis, medical image reconstruction, and weather forecasting, resulting in an expressive gain over the CPUs. This has been possible due to the development of the generalpurpose computing on graphics processing units (GPGPU), making them even more flexible through their high parallelism and adaptable application programming.

As a result of these technological advances, volume visualization techniques have also evolved considerably over the last years. Real-time volume rendering accelerated through GPUs has become an effective tool for volumetric data visualization and analysis.

This work presents a methodology for accelerating on GPUs a volume visualization technique for isosurface extraction, called marching cubes. The performance of the method is improved by means of auxiliary spatial data structures. Experimental results obtained on several volumetric datasets demonstrate the effectiveness of the proposed method. 
The contributions of this work are a comparative performance analysis of the marching cubes technique with different spatial data structures, and a development of an application capable of manipulating datasets from several fields of knowledge, which permits its use as a framework to be integrated in visualization environments, obtaining high real-time rendering rates as well.

This paper is organized as follows. Section 2 briefly reviews some relevant concepts of volume visualization and isosurface extraction, as well as the marching cubes algorithm and some spatial data structures used to improve the performance of the algorithm. Section 3 presents a methodology for speeding up the marching cubes technique on GPU. Section 4 describes and discusses the experimental results obtained by applying the proposed method to a number of volumetric datasets. Section 5 concludes the paper with some final remarks.

\section{Related concepts and work}

This section describes an overview of the volumetric visualization, followed by concepts of isosurface extraction, marching cubes algorithm, and a description of some data structures used to improve the performance of this algorithm.

\subsection{Volumetric visualization}

Volumetric visualization $[11,19,30]$ consists of a set of techniques used to study objects and natural phenomena from various fields of knowledge, such as biology, medicine, meteorology, oceanography, microscopy, geology, astronomy. The basic idea of these techniques is to perform a twodimensional projection (usually on a computer screen) from these volumes.

The volumetric data is usually represented by a set of volume elements, called voxels, where each one contains a specific value in a regular grid contained in the three-dimensional space. A voxel can be defined by a tuple $\langle x, y, z, S\rangle$, which represents the value $S$ associated to some property of a volume data, located at a 3D grid position $(x, y, z)$.

Volumetric visualization algorithms can be classified into two categories [11]: direct volume rendering (DVR) and surface-fitting (SF). The first one is characterized by the direct element mapping onto the screen space, without the use of geometric primitives as an intermediary representation, whereas the second consists of stages of feature extraction and representation of isosurfaces (surfaces that represent a set of points with the same scalar value), which are later rendered for visualization. These isosurfaces can be defined from surface primitives (such as polygons) or by a certain threshold.
Examples of DVR techniques include raycasting [25,27], splatting [42,47], cell-projection [48,49] and shear-warp [22, 26]. Examples of SF techniques are contour connection [20, $34]$ and marching cubes [14,24,29,39,46].

\subsection{Isosurface extraction}

An isosurface can be defined as a set of points that have the same value (called isovalue) in a volume data, that is, $\left\{\langle x, y, z\rangle \in \mathfrak{R}^{3}: f(x, y, z)=h\right\}$, for a grid position $(x, y, z)$ and some isovalue $h \in \Re$. The isosurface extraction process involves the generation of meshes (usually triangular) that approximately represents a certain surface. In the medical field, for instance, this procedure is commonly used in the visualization of organs, tissues and anatomic structures.

A very known isosurface extraction technique is the marching cubes algorithm [9, 14,29,33,46], which was originally developed to improve the study of 3D medical images. Later, many researches were conducted to optimize this technique through the use of spatial data structures to improve the processing of volume data. However, with the advent of modern graphics cards, techniques that take most advantages from the graphics hardware have been explored due to the high degree of parallelism present in these cards.

In relation to isosurface extraction approaches in GPU, Reck et al. [40] and Buatois et al. [3] proposed methods for extraction from unstructured tetrahedral meshes. Tatarchuk et al. [44] showed an implementation of a hybrid method that employs both marching cubes and marching tetrahedra [4, 15] techniques, using geometry shaders in GPU. Martin et al. [31] developed a technique to efficiently distribute all the work load of the isosurface extraction procedure among GPU resources in a cluster.

Pascucci [38] proposed a pipeline for the isosurface extraction procedure in which most of the stages is done in GPU, assigning only to the CPU the tasks of accessing the volume data and sending a set of vertices (corresponding to the volume cells) for the GPU. In his proposal, the GPU does not have access to the volume dataset. Therefore, the CPU is responsible for transmitting all kind of relevant information about the vertices.

Ciznicki et al. [6] presented an isosurface extraction approach for CT and MRI images that combines the marching tetrahedra algorithm with histogram pyramids [10,50] using multiple GPUs. Using a single GPU, their application provided a speedup of 107 times comparing to a standard CPU version. With four GPUs, it achieved a speedup of 3.3 times, in relation to the single GPU version.

Schindler et al. [41] proposed an adaptation of the marching cubes algorithm called marching correctors, which extracts isosurface from smoothed particle hydrodynamics (SPH) datasets. The GPU is responsible for computing the selection of seed cells of the datasets, which is the 


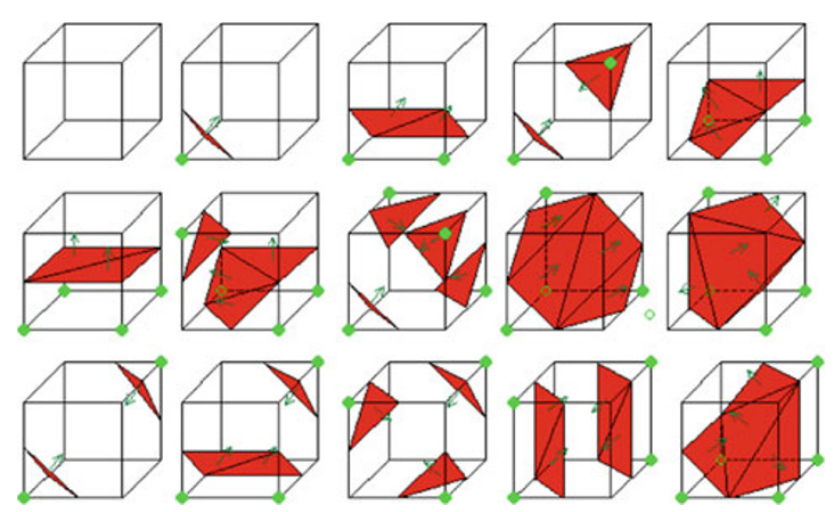

Fig. 1 Illustration of the 15 basic cases of the marching cubes technique. The green vertices are the ones classified as "inside" the isosurfaces, whereas the remaining as "outside" them. Image extracted from [29]

most expensive part of the proposed algorithm, achieving a performance gain of two orders of magnitude, comparing to other approaches that handle SPH datasets.

\subsection{Marching cubes}

The marching cubes technique uses a divide-and-conquer approach in which the volume data is processed through their cells (voxels), that are equivalent to cubes. In each cell, the intersection between its respective edges and the isosurface is verified. The values of each vertex cells are then compared to a given isovalue $h$ and these vertices are classified as "inside" or "outside" the isosurface. The first case is applied when the value of the vertex is greater than or equal to $h$ and the second one when it is less than $h$. Once defined the type of intersection, an approximation of the isosurface contained in the cell is done by constructing triangles.

As each of the 8 vertex cells has only two possible states, there is a total of $2^{8}=256$ cases of intersection between isosurface and cell edge, which are listed in a lookup-table. However, some pairs of these cases are symmetric or complementary to each other, which restrains the problem to 15 cases, as illustrated in Fig. 1. A demonstration of the marching cubes algorithm using different isovalues is shown in Fig. 2.

The pseudocode of the marching cubes technique can be briefly described according to the Algorithm 1. The advantage of this algorithm is that the processing of a cell is independent of the other ones, which allows its parallelization. However, as a disadvantage, it may generate holes in the isosurfaces, due to topological ambiguities of the cases.

Algorithm 1 runs in time $O(n)$, where $n$ is the total number of processed cells. On the other hand, many of these cells are empty, incurring an unnecessary waste of time. To minimize this waste, some spatial data structures are used to only process the active cells, that is, the ones that are
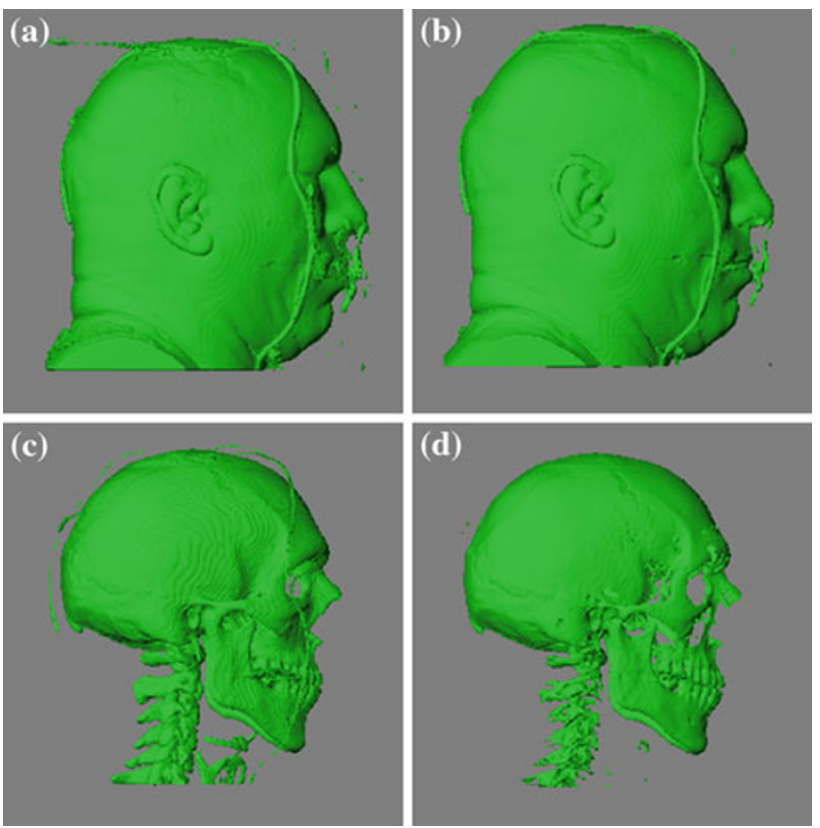

Fig. 2 Volume rendering of a male head dataset using marching cubes with different isovalues: a 30, b 50, c 75, d 100

intercepted by an isosurface, reducing the complexity of the algorithm.

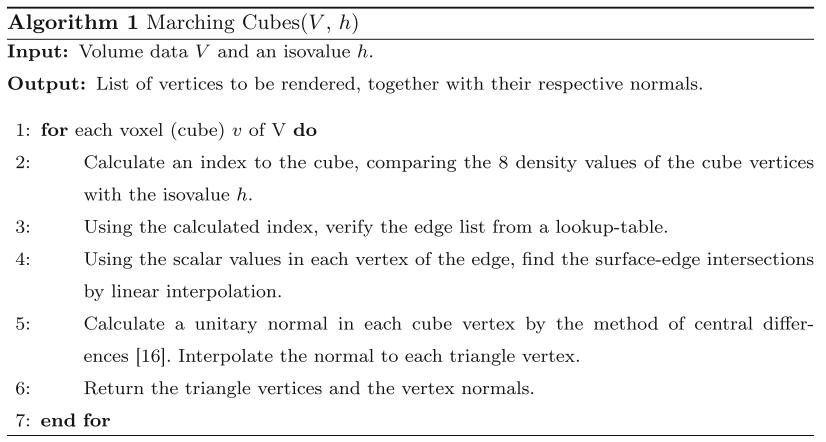

In the context of the acceleration of marching cubes algorithm on the GPU, Johansson and Carr [18] conducted a comparative analysis of its execution using data structures, such as k-d trees [2] and interval trees [5], reporting the rendering rate speedups obtained in relation to the $\mathrm{CPU}$. Their approach is based on a caching cell topology technique, which stores the 15 cases of marching cubes in GPU (once it can cache geometry in a very efficient way) and improves the performance of the case classification stage by means of an optimization of the pre-computation step on the CPU, making use of the span-space properties. The method achieves a maximum speedup (between the GPU acceleration with an interval tree and its CPU counterpart) of 4.3 times. However, the authors do not make experiments with larger datasets due to hardware limitations.

Goetz et al. [13] optimized the marching cubes algorithm using a vertex shader, achieving an interactive isosurface 
reconstruction, but their approach does not use any accelerating data structures to improve the overall performance of the algorithm, obtaining a maximum speedup of approximately 2.0 times.

Newman and Yi [32] developed an in-depth research about the possibilities of developing the marching cubes technique, describing their respective properties, extensions and attempts to solve its limitations. However, the paper only shows the differences between these possibilities in terms of algorithm complexity as well as visualization results, and does not make an analysis with volume datasets.

Smistad et al. [43] presented an implementation of the marching cubes algorithm written in OpenCL [37] that runs entirely in the GPU. Like [6], it uses the idea of histogram pyramids to generate the output stream of vertices to be rendered and runs as fast as CUDA and shader implementations, providing a very efficient storage scheme as well. However, the drawback of their implementation lies on the interoperability between OpenCL and OpenGL.

Concerning recent applications that make use of the marching cubes algorithm in GPU, Dembogurski et al. [7] used a marching cubes histogram pyramid implementation for a procedural terrain generation; Donlon et al. [8] accelerated the visualization and quantification of MRI datasets to help in the treatment of patients that suffer from rheumatoid and psoriatic arthritis; Kim et al. [21] presented a method for computing the surfaces of a protein molecule in interactive time; Lang et al. [23] developed an environment for fast and automated analysis of large SBFSEM (serial blockface scanning electron microscopy) datasets to extract neuron morphologies.

\subsection{Accelerating data structures}

There are several classes of data structures that are very useful to avoid the processing of empty cells. One of them consists of interval-based representations, which uses cell intervals to group cells [32]. The advantage of this type of representation is in its flexibility, being applied not only on regular grids, but also on non-regular grids, once it works from an interval space, instead of using the mesh space itself.

The main methods of this class are based on a representation called span-space [28], where each cell of the volume data is mapped to a two-dimensional point, whose coordinates $x$ and $y$ correspond, respectively, to the minimum and maximum values among the eight vertices that constitute a cell. From a given isovalue $h$, the points of the span-space that represent the active cells are the ones where $x \leq h$ and $y \geq h$.

A general scheme of the span-space is shown in Fig. 3. The blue area corresponds to the active cells of a volume data and the yellow areas to the cells that are not rendered, due to the fact that $x>h$ (yellow area located right of the blue

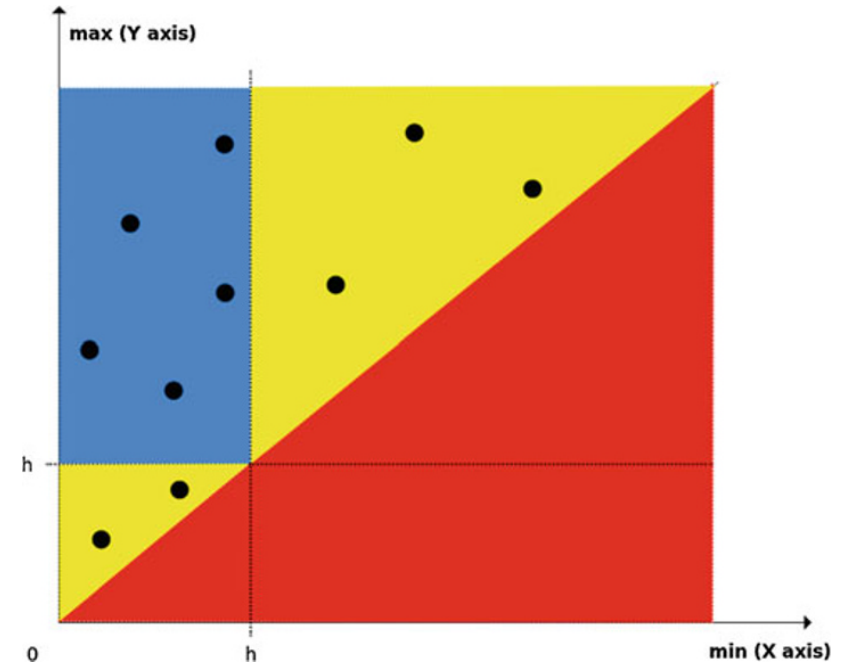

Fig. 3 Representation of the span-space proposed by Livnat et al. [28]

area) or $y<h$ (yellow area located below the blue area). No cell can be mapped to the red area, once $x$ is never greater than $y$.

This section describes some spatial data structures and how they can be used to improve the performance of the marching cubes algorithm.

\subsection{1 k-d tree}

The k-d tree [2] is a special case of the binary search tree, used to organize points located in a k-dimensional space. Each non-leaf node represents a splitting hyperplane that divides the space into two parts in a specific direction, which is defined according to the depth of this node in the tree. The left subtree contains all the points located at the left of the hyperplane and the right subtree contains the ones to the right. The leaf nodes store one point each.

In the marching cubes algorithm, the volume data is mapped onto a span-space before constructing the tree, once the queries in the $\mathrm{k}-\mathrm{d}$ tree are faster when working with points in a $2 \mathrm{D}$ plane rather than in a 3D space. Furthermore, every node stores a point in the span-space, instead of storing the points only in the leaves. The construction takes $O(n \log n)$ time, where $n$ is the total number of cells in the volume data, and demands a storage space of $O(n)$.

When searching in the tree, given an isovalue $h$, it will traverse only the nodes that correspond to the active cells of the volume data. Thus, the query takes $O(\sqrt{n}+p)$ time, where $p$ is the number of active cells.

\subsubsection{Interval tree}

The interval tree [5] is an ordered tree used to store intervals of values in 1D. Similarly to the k-d tree, it is an extension 
of the binary search tree, and allows an efficient search of all intervals that overlap with a given interval or point.

The root of the tree stores a value that corresponds to the median of the endpoints of all intervals and a list of intervals that contain this value. The left subtree stores the intervals that are completely below the median and the right subtree stores the ones completely above the median. Then, the process is repeated recursively for each subtree. It takes a construction time of $O(n \log n)$ and, like the k-d tree, a storage space of $O(n)$.

The span-space is suitable for constructing an interval tree. In this case, the intervals correspond to the volume cells, and the endpoints are the minimum and maximum values of the cell, which stands for its coordinates in the span-space.

Searching in an interval tree takes $O(\log n+p)$ time, where $p$ is the number of active cells, which makes it more efficient than the k-d tree. On the other hand, it demands higher memory space.

\subsubsection{Quadtree and octree}

A quadtree [12] is a tree data structure where every nonleaf node has exactly four children. It is used to partition a region in a $2 \mathrm{D}$ space into four equal regions (or quadrants). These regions are then partitioned into other four subregions, and so on, until the subregion is empty, which characterizes a leaf node. The 3D analogous structure of a quadtree is called octree, which partitions a 3D space region into eight subregions (or octants).

Once the span-space is a 2D space, it can be represented by a quadtree. Let $l$ be the number of bits used to store the volume data values, which means that there are $2^{l}$ possible values (ranging between 0 and $2^{l}-1$ ) for a vertex cell. Thus, the span-space is a $2^{l} \times 2^{l}$ region, and the points correspond to the mapped cells. Every node in the tree stores the information of a point in the span-space.

However, more than one cell can be mapped to a same point in the span-space. To overcome this problem, each node in the quadtree also stores a pointer to a list of the volume data cells that were mapped to this point. Then, the queries can be made as usual, traversing the nodes corresponding to the active cells.

In the octree case, the tree is built directly from the volume data. However, in case of non-regular grids (i.e., when volume dimension is not a power of two), the subregions have different sizes, once we are partitioning cell regions.

\section{Methodology}

The significant evolution of the programmability of the graphics hardware has allowed the isosurface extraction procedure to be accelerated by the GPU, taking advantage of its parallel architecture. However, the bus used to establish a communication between the CPU and the GPU is a bottleneck for this acceleration, which means that transferring all the tasks to the GPU may not be the best solution. Thus, in order to maximize the performance of this procedure, an adequate planning of the graphics pipeline is needed, picking up the tasks that can be run on the CPU and the ones that can be transferred to the GPU, as well as a proper use of all the memory hierarchy. Some proposals of graphics pipeline for isosurface extraction were made by Buatois et al. [3], Johansson [17], Martin et al. [31], Pascucci [38], Reck et al. [40], and Tatarchuk et al. [44].

The methodology proposed in this work is restricted to the marching cubes technique, rather than generalizing to the isosurface extraction process. Figure 4 shows a general scheme, composed of six stages. At the Stage 1, the CPU reads a volume dataset of dimensions $N_{x} \times N_{y} \times N_{z}$, which is then allocated both in the main memory (RAM, used by the CPU) and in the video memory (VRAM, used by the GPU). Except for the octree, an extra memory space is allocated for the span-space related to the volume data.

Later, one of the spatial data structures described in Sect. 2.4 is constructed from the volume data and stored only in the main memory (stage 2 ). Then, the volume data is freed from the main memory, but it remains allocated in the video memory.

Algorithm 2 details the two aforementioned stages of the scheme. Once the preprocessing stage is done, the marching cubes algorithm is started (stage 3). From an isovalue $h$ specified by the user, the CPU performs a search in the data structure, traversing only the nodes that correspond to the active volume cells, and creating a list of these cells, which is then transferred to the GPU via communication bus.

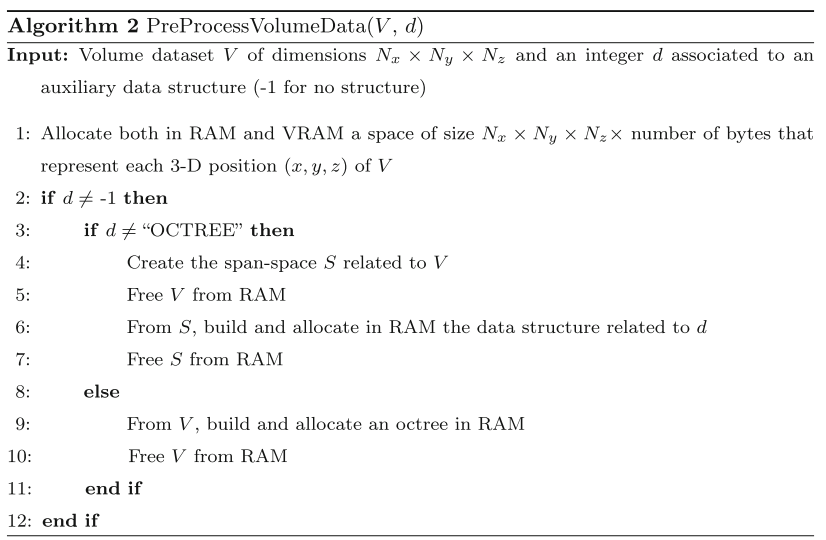

With the list of active cells and the isovalue $h$, the marching cubes algorithm then proceeds on the GPU. Each cell is classified into one of the 15 cases of marching cubes (shown in Fig. 1) by comparing $h$ to the eight cell vertices. From this comparison, a cell index is created and then used to define the number of vertices needed to render the isosurface contained 


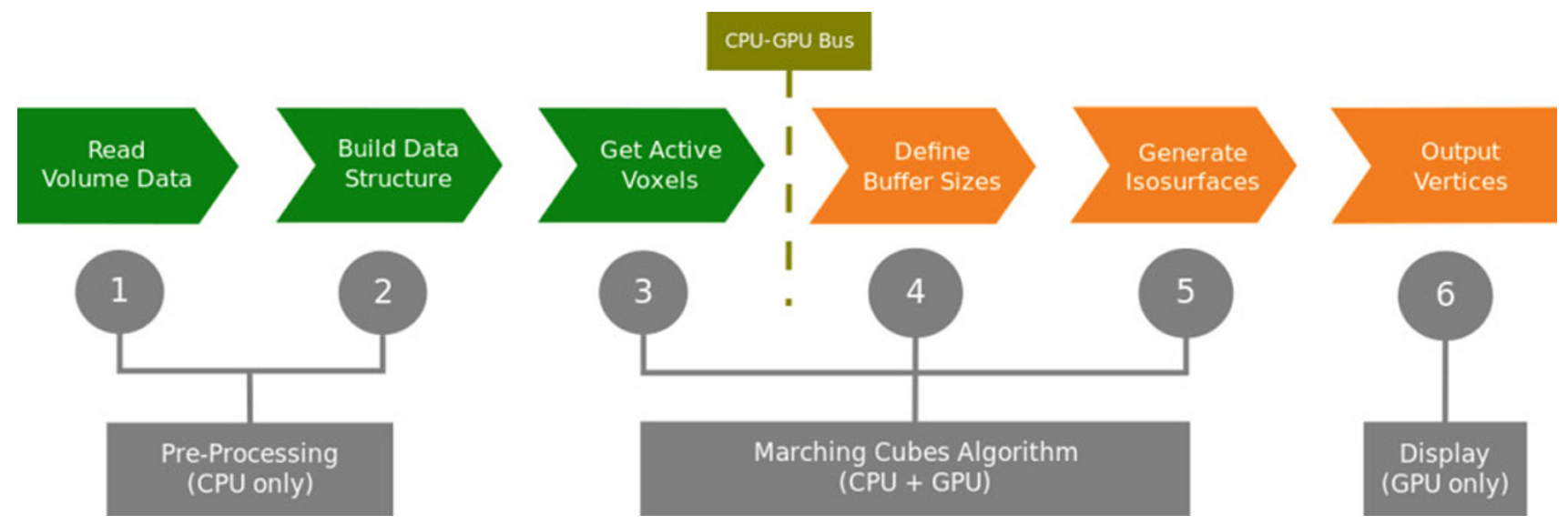

Fig. 4 Our method for acceleration of the marching cubes algorithm. Stages 1 and 2 are responsible for the preprocessing stage; stages 3 to 5 correspond to the execution of marching cubes and stage 6 makes the display of vertices on the screen. Green boxes stand for the actions executed on CPU, whereas the orange boxes the ones on GPU

In addition to the algorithms that are responsible for the execution of the marching cubes technique, some of the GPU inherent resources were employed in order to improve the rendering rate of the volume data. Among these resources are the use of texture memory, which is faster for handling read-only data, to store the lookup-tables employed by the marching cubes algorithm and the volume data itself, and the use of shared memory, which permits the data sharing among threads contained in a same block, besides being faster than the standard video memory. In the GPU marching cubes algorithm, the shared memory is used to compute, for each active cell, the intersections between an isosurface contained in the cell and the cell edges.

The pseudocodes of the following algorithms were adapted from [36]. Before the execution of these algorithms, the respective number of blocks (as well as their sizes) used to help on the parallelization of their procedures are determined.

Algorithm 3 represents the first step of the execution of marching cubes in GPU (stage 4 of Fig. 4). The goal of this algorithm is to calculate the exact amount of video memory that will be used to store the vertices and the normals related to the volume data. Once this amount is obtained, the GPU allocates the appropriate space in the vertex buffers, which will then store the vertices to be output and their respective normals.

Once this procedure is done, the Algorithm 4 is started. It is important to point out the repetition of the calculation of the cell indexes (line four of the Algorithms 3 and 4), which in GPU is faster than storing the results provided by Algorithm 3 in an array and retrieving them in the subsequent algorithm. Later, the vertex and normal buffers (called $P$ and $N$, respectively) are filled and the volume data is visualized, thus finishing the steps of the marching cubes technique. 

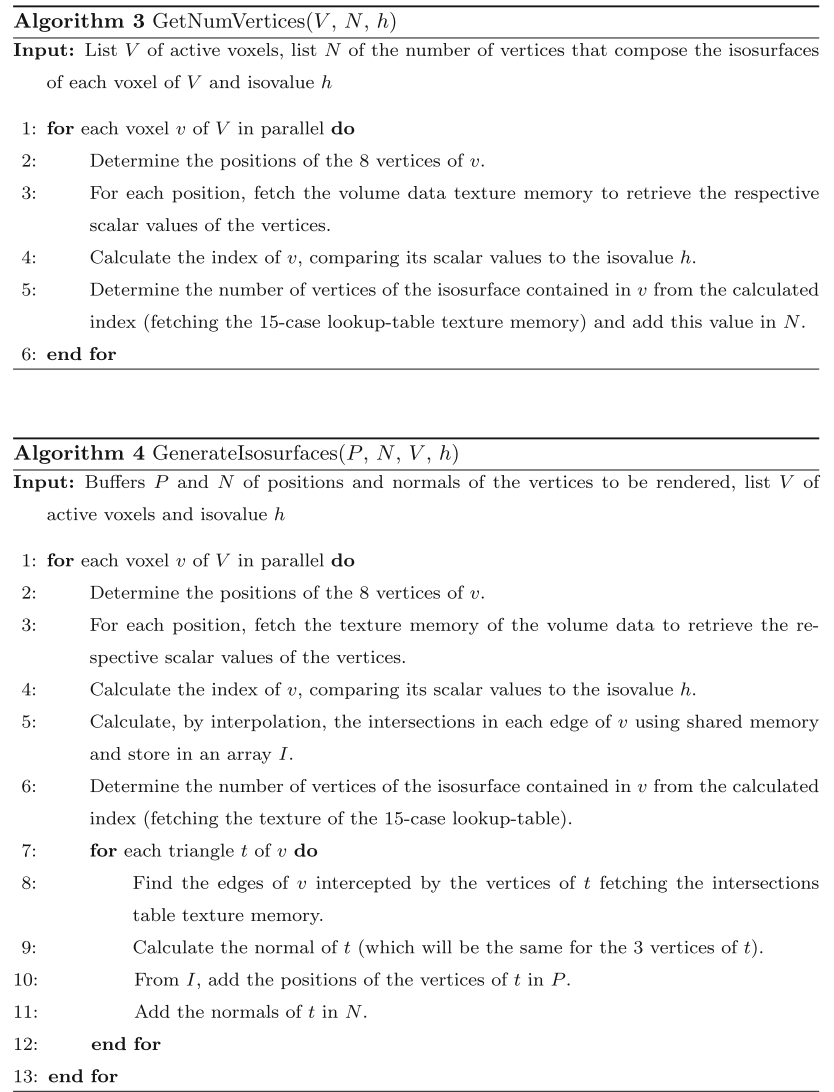

\section{Experimental results}

The tests were executed on an AMD Phenom II X6 1090T $3.2 \mathrm{GHz}$ processor with $8 \mathrm{~GB}$ of RAM, and an NVIDIA GeForce GTS 450 with 1 GB of VRAM (together with its most recent drivers), using a C-like programming language, OpenGL 4.2 and CUDA 4.1 APIs.

The experiments were made using 8-bit datasets from [1] and [45], where each scalar value ranges from 0 to 255 . Table

Table 1 List of volume datasets with their respective sizes in voxels, isovalues, number of triangles and memory space allocated during execution of marching cubes algorithm (in megabytes)

\begin{tabular}{llrrr}
\hline Volume name & Dimensions & Isovalue & \multicolumn{2}{c}{ \# Triangles Space (MB) } \\
\hline Fuel & $64 \times 64 \times 64$ & 10 & 11,534 & 0.51 \\
Hydrogen atom & $128 \times 128 \times 128$ & 20 & 47,864 & 3.09 \\
Angiography & $256 \times 320 \times 128$ & 80 & 84,974 & 11.94 \\
Engine & $256 \times 256 \times 128$ & 155 & 207,592 & 12.75 \\
Lobster & $301 \times 324 \times 56$ & 45 & 317,024 & 12.46 \\
Head & $128 \times 256 \times 256$ & 50 & 544,588 & 20.46 \\
Bonsai & $256 \times 256 \times 256$ & 45 & 858,118 & 35.64 \\
Knee & $379 \times 229 \times 205$ & 60 & $1,027,838$ & 40.49 \\
Sheep heart & $352 \times 352 \times 256$ & 80 & $1,397,064$ & 61.87 \\
Aneurism & $512 \times 512 \times 512$ & 45 & 835,068 & 147.11 \\
\hline
\end{tabular}

1 shows a list of volume datasets used in the tests, together with their respective dimensions (in voxels), isovalues (input to the marching cubes algorithm), number of triangles rendered in the screen (which depends on the isovalue), and memory space allocated during the execution of the marching cubes algorithm, which corresponds to the size of the volume datasets ( 1 byte per voxel) plus the amount of the vertex and normal buffers ( 4 bytes per vertex and normal, which leads to a total of 24 bytes per triangle, once there are two buffers). The isovalues for "Fuel", "Hydrogen atom" and "Engine" datasets were the same as those used by [18], so that a more accurate comparison can be made. The results of the rendering of each dataset, made from the application described in this work, are shown in Fig. 5.

Figure 6 shows two plots that illustrate the average time (in milliseconds) of 50 executions of the marching cubes implementation on the GPU for different block size values, using each of the volume datasets and their respective isovalues listed in Table 1. The plot (a) stands for the executions of a brute force implementation of the marching cubes algorithm that runs without the aid of accelerating data structures and sequentially traverses all voxels of a volume dataset (including the empty ones), and (b) the executions using an interval tree to store the voxels from the datasets. From both plots, it can be noticed that for block sizes of 64 and higher, the running time of the marching cubes increases very slightly, and it does not rely on whether a data structure is used or not for acceleration. In other words, for any data structure used in the marching cubes algorithm, the behavior of the curves in the plot will be the same. As mentioned in Sect. 3, although a higher number of threads means higher parallelization, the time spent to create the threads also gets higher, hence stabilizing the performance. Thus, all the tests were run with a block size value of 64 .

Table 2 shows the average frame rate of the execution of marching cubes algorithm in CPU and GPU, for all data structures described in Sect. 2.4, and comparing to their respective CPU versions of the algorithm. These results do not consider the time spent on the pre-processing stages (volume data reading and data structure construction), regarding only the events that occur between the search in the data structure and the volume display on the screen (Stages 3-6 of Fig. 4).

As it is possible to observe, the interval tree provided the best results (highlighted in bold in Table 2), not only among all the data structures used in the tests, but also in the acceleration factor compared to the CPU marching cubes, achieving a maximum speedup of 18.2 times for the "Aneurism" dataset. This result was expected because the interval tree has asymptotically a better query time than the other structures. Furthermore, the obtained speedups are superior than those of Johansson and Carr's approach [18], which achieves a maximum speedup of 4.3 times (for the "Hydrogen Atom" dataset and the interval tree) using pre-calculated normals, 

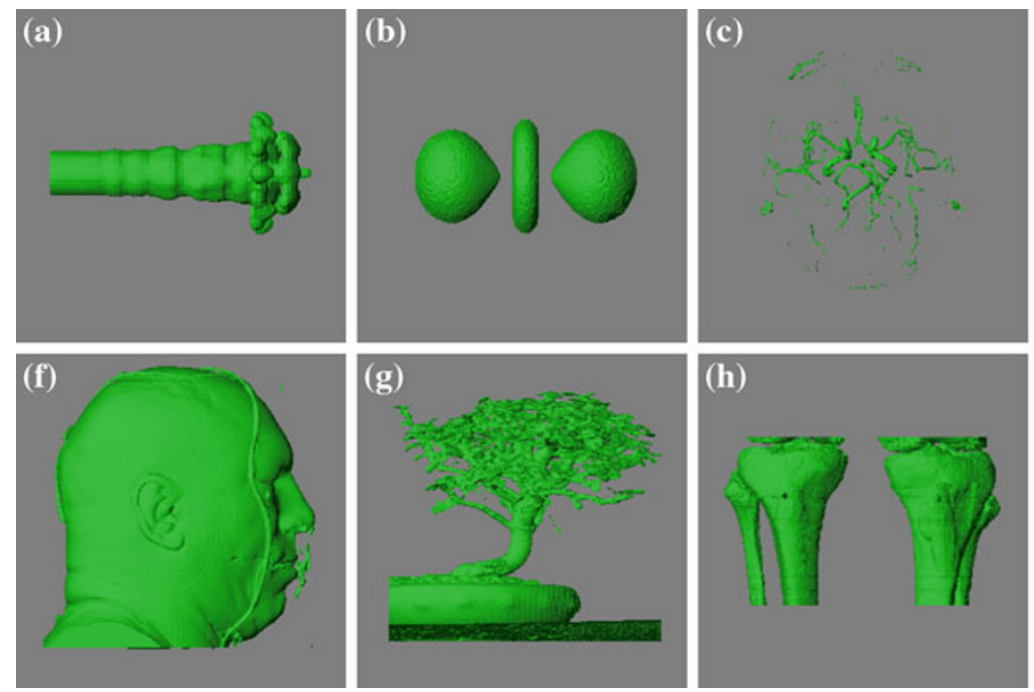
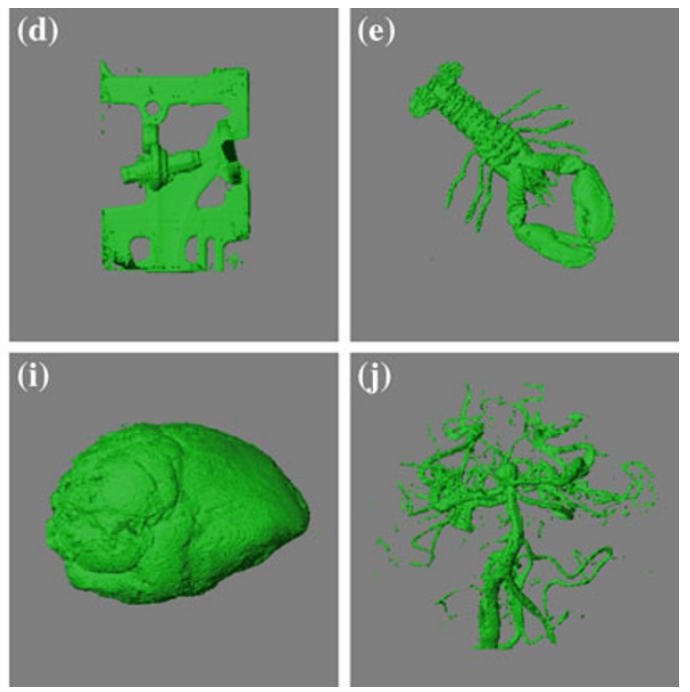

Fig. 5 Rendering results of each dataset generated by the application, with the isovalues listed in Table 1. a Fuel, b hydrogen atom, c angiography, $\mathbf{d}$ engine, $\mathbf{e}$ lobster, $\mathbf{f}$ head, $\mathbf{g}$ bonsai, $\mathbf{h}$ knee, $\mathbf{i}$ sheep heart, $\mathbf{j}$ aneurism

Fig. 6 Average times of executions of the marching cubes algorithm on GPU for different block sizes. Graph a shows the results for the brute force version and $\mathbf{b}$ for the interval tree
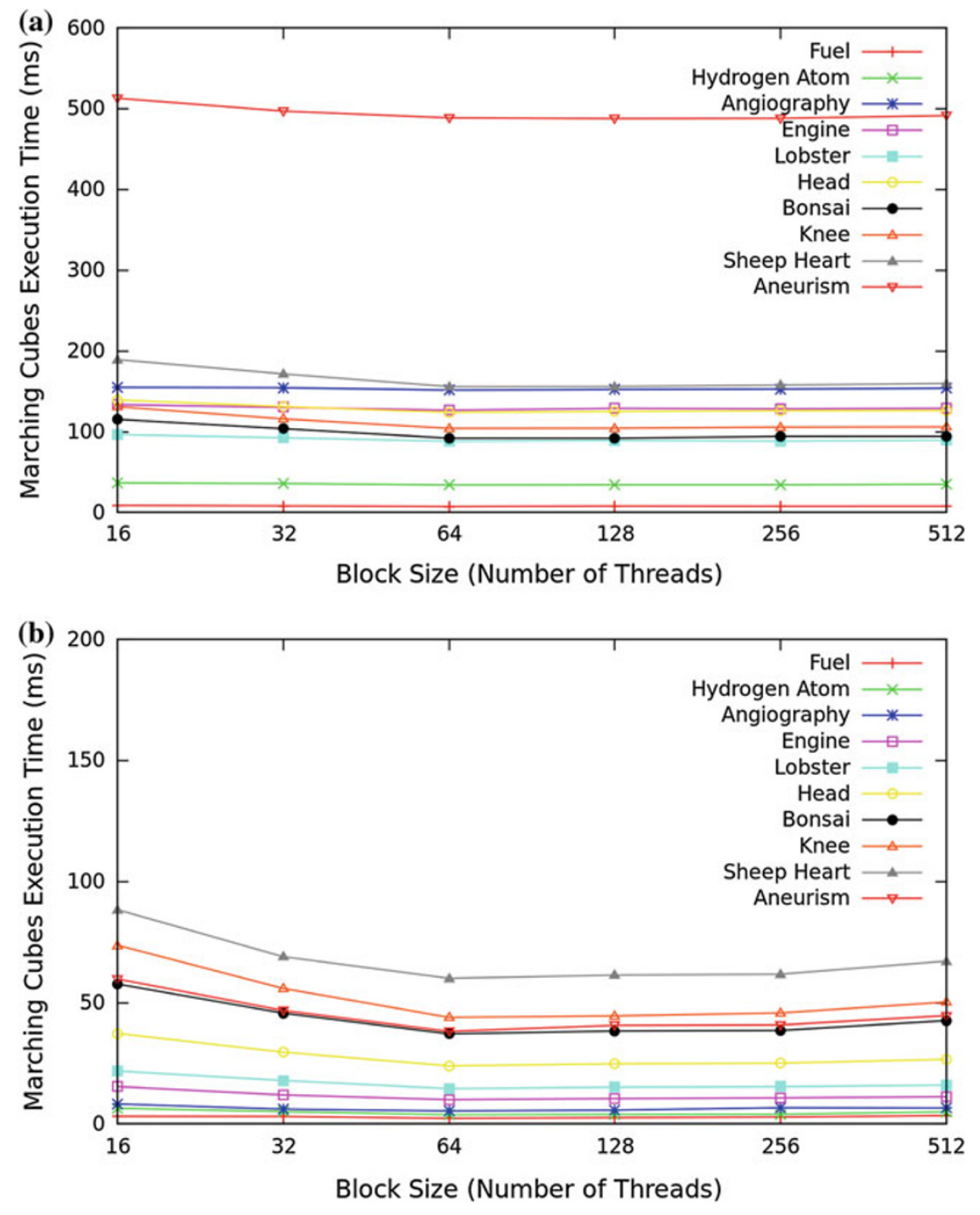
Table 2 Average frame rate (in frames per second) of the execution of marching cubes algorithm in CPU and GPU, with different data structures, comparing against the brute force version

Values between parenthesis correspond to the acceleration factor related to their respective data structure versions of marching cubes in CPU for each volume dataset

\begin{tabular}{|c|c|c|c|c|c|}
\hline Volume name & Brute force & $\mathrm{k}$-d tree & Interval tree & Quadtree & Octree \\
\hline \multicolumn{6}{|l|}{ CPU } \\
\hline Fuel & 83.3 & 105.6 & 114.4 & 108.0 & 98.3 \\
\hline Hydrogen atom & 17.5 & 23.4 & 25.6 & 24.0 & 19.2 \\
\hline Angiography & 7.5 & 12.9 & 14.1 & 13.6 & 10.7 \\
\hline Engine & 4.6 & 5.2 & 5.7 & 5.5 & 4.8 \\
\hline Lobster & 3.2 & 3.7 & 4.1 & 3.9 & 3.6 \\
\hline Head & 1.8 & 2.0 & 2.3 & 2.1 & 1.9 \\
\hline Bonsai & 1.2 & 1.3 & 1.6 & 1.4 & 1.3 \\
\hline Knee & 1.1 & 1.2 & 1.4 & 1.3 & 1.1 \\
\hline Sheep heart & 0.8 & 0.9 & 1.0 & 0.9 & 0.8 \\
\hline Aneurism & 0.6 & 0.9 & 1.3 & 1.2 & 0.7 \\
\hline \multicolumn{6}{|l|}{ GPU } \\
\hline Fuel & $195.4(2.3)$ & $220.7(2.1)$ & $246.4(2.2)$ & $229.9(2.1)$ & $205.7(2.1)$ \\
\hline Hydrogen Atom & $77.0(4.4)$ & $142.8(6.1)$ & $165.0(6.4)$ & $149.5(6.2)$ & $96.5(5.0)$ \\
\hline Angiography & $24.7(3.3)$ & $109.6(8.5)$ & $133.7(9.5)$ & $89.2(6.6)$ & $58.7(5.5)$ \\
\hline Engine & $26.6(5.8)$ & $61.0(11.7)$ & $81.1(14.2)$ & $56.8(10.3)$ & $38.4(8.0)$ \\
\hline Lobster & $30.1(9.4)$ & 42.8 (11.6) & $58.5(14.3)$ & 29.8 (7.6) & $27.4(7.6)$ \\
\hline Head & $17.9(9.9)$ & $22.7(11.4)$ & $35.8(15.6)$ & $28.9(13.8)$ & $20.9(11.0)$ \\
\hline Bonsai & 10.7 (8.9) & $13.2(10.2)$ & $25.9(16.2)$ & $18.7(13.4)$ & $11.5(8.8)$ \\
\hline Knee & $8.2(7.4)$ & $9.7(8.1)$ & $22.4(16.0)$ & $10.7(8.2)$ & $9.1(8.2)$ \\
\hline Sheep Heart & $6.3(7.9)$ & $7.4(8.2)$ & $16.3(16.3)$ & $8.9(9.9)$ & $6.6(8.3)$ \\
\hline Aneurism & $2.1(3.5)$ & $10.2(11.3)$ & 23.7 (18.2) & $11.9(9.9)$ & $9.5(13.5)$ \\
\hline
\end{tabular}

while the method proposed in this paper calculates them onthe-fly.

Another important fact to be pointed out is the performance of quadtree, which obtained a better frame rate than the k-d tree and the octree, standing only behind the interval tree. It achieved a maximum speedup of 13.8 times for the "Head" dataset. For the other datasets, excluding the "Fuel", the speedup was between 6.2 and 10.3 .

Concerning the building time of the data structures, quadtree had a much higher performance than the other ones, as shown in Fig. 7. For all datasets used in the tests, the building time of the quadtree was very much smaller than the other data structures, not having a significant gain of time as the volume datasets get larger, which is very efficient for real applications that process great amounts of volume data. For the rest of the data structures, the building time increases considerably among the datasets.

Octree provided the second best building times. Even though its building algorithm is similar to the one of quadtree, the fact that it is applied in the 3D space brings on a higher waste of time, once the total number of subdivisions is higher. In third place, comes the k-d tree, which has a worse building time than the octree because of the successive executions of the algorithm that finds the median of the points located in the span-space. Finally, interval tree achieved the worst building times among the tested data structures. It happened due to the time spent in sorting the cell lists, which has a higher time complexity than finding a median.

The bottleneck of this approach resides on the fact that the searches in the data structures are done on the CPU. It is feasible to implement all of the data structure algorithms on the GPU, as well as storing the data structure itself in the video memory, but since most of the tree search algorithms are recursive and CUDA does not support recursions, if nonrecursive versions of these algorithms were implemented on the GPU, there would have a large waste of time and space to create stacks and loops used to simulate the eventual recursive calls.

\section{Conclusions and future work}

Volume visualization techniques allow users to explore and analyze tridimensional data, which benefit several knowledge domains, such as biology, medicine, meteorology, oceanography, geology. A challenging task of volume visualization is the development of efficient algorithms for representing, manipulating and rendering complex and large datasets. 
Fig. 7 Average building times of the data structures for each volume dataset

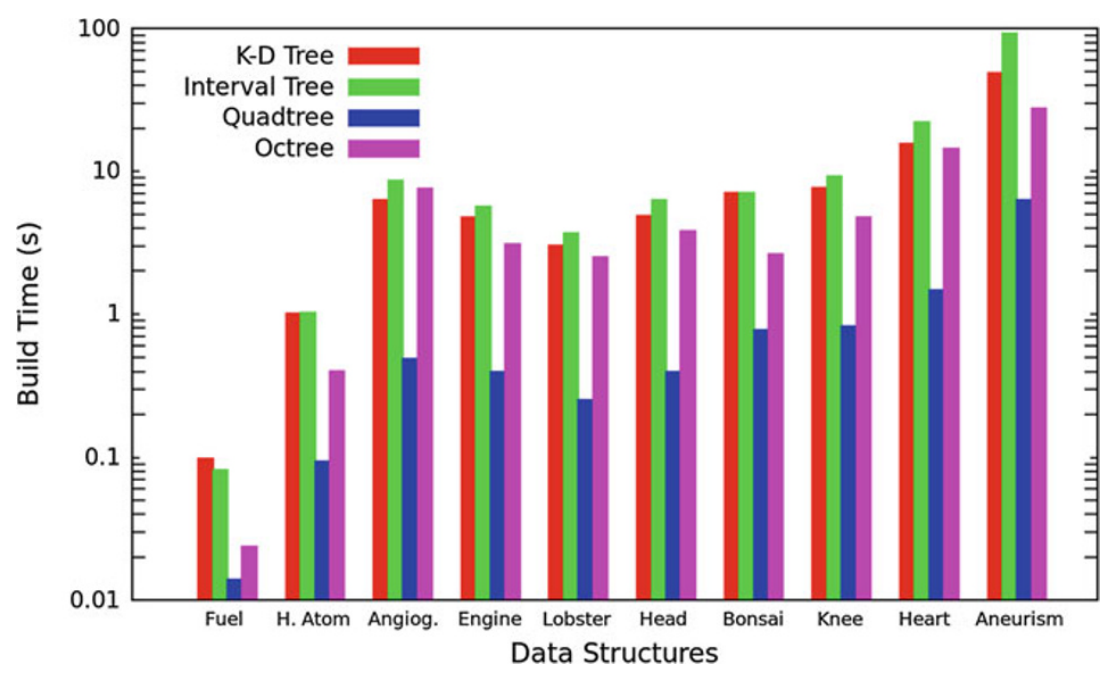

This paper described a comparative analysis among four different spatial data structures for speeding up the marching cubes algorithm through graphics processing units, together with an approach using CUDA framework. Time complexity of the algorithm depends on the volume data cells that contain an isosurface, instead of the total number of cells, avoiding the processing of empty cells.

Experimental results obtained from several volumetric datasets demonstrate that it was possible to accelerate the marching cubes algorithm by a factor of approximately 18 times when compared to the CPU approach.

Among the four data structures described in this paper, interval tree provided the best rendering rates, but the time spent in building the structure was relatively high. On the other hand, quadtree achieved very satisfactory building times, even for large volume datasets, besides slightly lower rendering rates compared to those of the acceleration with an interval tree. However, since the building of a data structure is done only once, while the search is performed whenever the volume needs to be rendered, it is more worthwhile to use the interval tree rather than the quadtree in this case.

Directions for future work include the implementation of the data structures mentioned in this paper on the GPU, together with their respective building and searching operations so that they can be done in parallel, and an extension of the proposed method to open frameworks, such as OpenCL [37], once that CUDA framework is restricted to NVIDIA [35] graphic cards. Furthermore, a case study with more powerful graphic cards (such as those from NVIDIA Kepler architecture) is desired as well.

Acknowledgments The authors are grateful to FAPESP, CNPq, and National Institute of Science and Technology in Medicine Assisted by Scientific Computing (INCT/MACC) for the financial support.

\section{References}

1. The volume library. http://lgdv.cs.fau.de/External/vollib

2. Bentley J (1975) Multidimensional binary search trees used for associative searching. Commun ACM 18(9):509-517

3. Buatois L, Caumon G, Lévy B (2006) GPU accelerated isosurface extraction on tetrahedral grids. In: Advances in visual computing. Lecture notes in computer science, vol 4291, Springer, Berlin, pp 383-392

4. Chan S, Purisima E (1998) A new tetrahedral tesselation scheme for isosurface generation. Comput Graph 22(1):83-90

5. Cignoni P, Marino P, Montani C, Scopigno R (1997) Speeding up isosurface extraction using interval trees. IEEE Trans Visual Comput Graph 3:158-170

6. Ciznicki M, Kierzynka M, Kurowski K, Ludwiczak B, Napierala K, Palczynski J (2011) Efficient isosurface extraction using marching tetrahedra and histogram pyramids on multiple GPUs. In: Proceedings of the 9th international conference on parallel processing and applied mathematics, Torun, Poland, pp 343-352

7. Dembogurski B, Clua E, Vieira M, Leta F (2008) Procedural terrain generation at GPU level with marching cubes. In: Proceedings of the VII Brazilian symposium of games and digital entertainmentcomputing track, Belo Horizonte, MG, Brazil, pp 37-40

8. Donlon B, Veale D, Brennan P, Gibney R, Carr H, Rainford L, Ng C, Pontifex E, McNulty J, FitzGerald O, Ryan J (2012) MRIbased visualisation and quantification of rheumatoid and psoriatic arthritis of the knee. In: Visualization in medicine and life sciences II, mathematics and visualization, Springer, pp 45-59

9. Dürst M (1988) Letters: additional reference to marching cubes. ACM Comput Graph 22(4):72-73

10. Dyken C, Ziegler G, Theobalt C, Seidel H (2008) High-speed marching cubes using histopyramids. Comput Graph 27(8):20282039

11. Elvins T (1992) A survey of algorithms for volume visualization. SIGGRAPH Comput Graph 26(3):194-201

12. Finkel R, Bentley J (1974) Quad trees: a data structure for retrieval on composite keys. Acta Inf 4(1):1-9

13. Goetz F, Junklewitz T, Domik G (2005) Real-time marching cubes on the vertex shader. In: Eurographics. Dublin, Ireland

14. Gong F, Zhao X (2010) Three-dimensional reconstruction of medical image based on improved marching cubes algorithm. In: Proceedings of the international conference on machine vision and human-machine interface, Kaifeng, China, pp 608-611 
15. Guéziec A, Hummel R (1995) Exploiting triangulated surface extraction using tetrahedral decomposition. IEEE Trans Visual Comput Graph 1(4):328-342

16. Jeffreys H, Jeffreys B (1988) Central differences formula. In: Methods of mathematical physics, Cambridge University Press, Cambridge, pp 284-286

17. Johansson $G$ (2005) Accelerating isosurface extraction by caching cell topology with graphics hardware. Master's thesis, University College Dublin, Ireland

18. Johansson G, Carr H (2006) Accelerating marching cubes with graphics hardware. In: Proceedings of the conference of the center for advanced studies on collaborative research. Toronto, ON, Canada

19. Kaufman A (1991) Volume visualization. IEEE Computer Society Press, Los Alamitos

20. Keppel E (1975) Approximating complex surfaces by triangulation of contour lines. IBM J Res Dev 19(1):2-11

21. Kim B, Kim K, Seong J (2012) GPU accelerated molecular surface computing. Appl Math Inf Sci 6(1S):185S-194S

22. Lacroute P (1996) Fast volume rendering using a shear-warp factorization of the viewing transformation. $\mathrm{PhD}$ thesis, Stanford University, Stanford, CA, USA

23. Lang S, Drouvelis P, Tafaj E, Bastian P, Sakmann B (2011) Fast extraction of neuron morphologies from large-scale SBFSEM image stacks. J Comput Neurosci 31(3):533-545

24. Lengyel E (2010) Transition cells for dynamic multiresolution marching cubes. J Graph GPU Game Tools 15(2):99-122

25. Levoy M (1990) Efficient ray tracing of volume data. ACM Trans Graph 9(3):245-261

26. Li T, Xie M, Zhao W, Wei Y (2010) Shear-warp rendering algorithm based on radial basis functions interpolation. In: Proceedings of the 2nd international conference on computer modeling and simulation, Sanya, China, pp 425-429

27. Liu B, Clapworthy G, Dong F (2009) Accelerating volume raycasting using proxy spheres. Comput Graph Forum 28(3):839-846

28. Livnat Y, Shen HW, Johnson R (1996) A near optimal isosurface extraction algorithm using the span space. IEEE Trans Visual Comput Graph 2(1):73-84

29. Lorensen W, Cline H (1987) Marching cubes: a high resolution 3D surface construction algorithm. Comput Graph 21(4):163-169

30. Lum EB, Wilson B, liu Ma K (2004) High-quality lighting and efficient pre-integration for volume rendering. In: Proceedings of the joint Eurographics-IEEE TVCG symposium on visualization, pp 25-34

31. Martin S, Shen HW, McCormick P (2010) Load-balanced isosurfacing on multi-GPU clusters. In: Proceedings of the Eurographics symposium on parallel graphics and visualization, Norrküping, Sweden, pp 91-100

32. Newman T, Yi H (2006) A survey of the marching cubes algorithm. Comput Graph 30(5):854-879

33. Nielson GM, Hamann B (1991) The asymptotic decider: resolving the ambiguity in marching cubes. In: Proceedings of the 2nd conference on visualization, San Diego, CA, USA, pp 83-91
34. Nurzyńska K (2009) 3D object reconstruction from parallel crosssections. In: Bolc L, Kulikowski J, Wojciechowski K (eds) Computer vision and graphics. Lecture notes in computer science, vol 5337, Springer, Berlin, pp 111-122

35. NVIDIA CUDA C programming guide version 3.2. http:// developer.download.nvidia.com/compute/cuda/3_2_prod/toolkit/ docs/CUDA_C_Programming_Guide.pdf

36. NVIDIA (2012) CUDA C/C++ SDK code examples. http:// developer.nvidia.com/cuda-cc-sdk-code-samples

37. OpenCL The khronos group. http://www.khronos.org/opencl/

38. Pascucci V (2004) Isosurface computation made simple: hardware acceleration, adaptive refinement and tetrahedral stripping. In: Proceedings of the joint Eurographics-IEEE TVCG symposium on visualization, Konstanz, Germany, pp 293-300

39. Pöthkow K, Weber B, Hege HC (2011) Probabilistic marching cubes. Comput Graph Forum 30(3):931-940

40. Reck F, Dachsbacher C, Grosso R, Greiner G, Stamminger M (2004) Realtime isosurface extraction with graphics hardware. In: Proceedings of the Eurographics 2004 short presentations and interactive demos, Grenoble, France, pp 33-36

41. Schindler B, Fuchs R, Waser J, Peikert R (2011) Marching correctors-fast and precise polygonal isosurfaces of SPH data. In: Proceedings of the 6th international smoothed particle hydrodynamics European research interest community (SPHERIC) workshop, Hamburg, Germany, pp 125-132

42. Schlegel P, Pajarola R (2009) Layered volume splatting. In: Advances in visual computing, lecture notes in computer science, vol 5876, Springer, pp 1-12

43. Smistad E, Elster AC, Lindseth F (2011) Fast surface extraction and visualization of medical images using openCL and GPUs. In: Proceedings of the joint workshop on high performance and distributed computing for medical imaging, Toronto, Canada

44. Tatarchuk N, Shopf J, DeCoro C (2007) Real-time isosurface extraction using the GPU programmable geometry pipeline. In: Proceedings of the ACM SIGGRAPH 2007 courses, San Diego, CA, USA, pp 122-137

45. Volvis: volume datasets. http://www.volvis.org

46. Wang Z, Fan B, Li N, Zhang H (2009) Iso-surface extraction and optimization method based on marching cubes. In: Proceedings of the international conference on semantics, knowledge and grid, Zhuhai, China, pp 458-460

47. Westover L (1990) Footprint evaluation for volume rendering. In: Proceedings of the 17th annual conference on computer graphics and interactive techniques, Dallas, TX, USA, pp 367-376

48. Wilhelms J (1990) A coherent projection approach for direct volume rendering. Technical report of University of California, Santa Cruz, CA, USA

49. Zhu S, Gu YL (2008) Volume rendering algorithm of irregular volume based on cell projection. Comput Eng Appl 44(15):68-70

50. Ziegler G, Tevs A, Theobalt C, Seidel HP (2006) On-the-fly point clouds through histogram pyramids. In: Proceedings of the 11th international fall workshop on vision, modeling and visualization, Aachen, Germany, pp 137-144 